\title{
Clonage et consensus : le projet de loi C-6
}

$\mathrm{L}$ a nouvelle annonçant que des chercheurs coréens ont réussi à cultiver des cellules souches embryonnaires par transfert de noyau d'une cellule somatique (TNCS) - une forme de clonage qui présente d'énormes possibilités pour le traitement des maladies chroniques et les remplacements d'organes - arrive au moment où les parlementaires canadiens se mettent d'accord sur un projet de loi qui interdirait ce genre de recherche au Canada (voir les pages 1086 et 1090). Au Canada, la législation réglementant les recherches sur les cellules souches accuse du retard par rapport à celle des États-Unis et de la Grande-Bretagne. Aux États-Unis, aucun financement de l'État ne peut servir à appuyer des recherches sur le TNCS ou la création d'embryons comme sources de cellules souches; l'État ne peut financer que des recherches sur des lignées de cellules souches existantes. Pour cette raison, les plus brillants scientifiques quittent les États-Unis pour aller travailler ailleurs, et certaines universités, comme Harvard, ont décidé de se priver de subventions fédérales afin de pouvoir échapper aux lois fédérales qui réglementent la recherche.

Au Royaume-Uni, la loi n'interdit pas l'utilisation d'embryons à des fins de recherche, ni les études sur le clonage thérapeutique comme celles qui ont lieu en Corée, compte tenu de leurs avantages potentiels pour l'humanité. Les scientifiques qui font des recherches sur les cellules souches doivent être autorisés par un organisme national et ils sont assujettis à sa réglementation. Toutefois, quelques activités, comme l'implantation d'embryons clonés dans un utérus humain, sont passibles de poursuites pénales.

Au Canada, les lois sur les technologies de reproduction évoluent de peine et de misère puisqu'elles doivent faire face, d'une part, à l'opposition éthique des personnes qui considèrent trop permissives les utilisations admissibles des embryons humains et, d'autre part, aux objections pragmatiques du milieu scientifique et médical pour qui la loi est trop restrictive. En ce moment même, un comité du Sénat a approuvé le projet de loi $\mathrm{C}-6^{1}$ sans modification, ce qui rend son adoption en troisième lecture presque certaine.

Le «vide législatif» qui existait pendant que le projet de loi C-6 et ses précurseurs avançaient difficilement au Parlement a eu un effet d'inhibition sur la recherche au Canada. Malgré les lignes directrices formulées par les Instituts de recherche en santé du Canada (IRSC), l'incertitude était suffisante pour immobiliser le financement de toute recherche sur le clonage thérapeutique. En vertu de la nouvelle loi (façonnée d'après les lignes directrices des IRSC), les chercheurs canadiens demeureront défavorisés dans un secteur concurrentiel. Il sera difficile d'attirer de jeunes scientifiques, de retenir les chercheurs déjà établis et d'obtenir des subventions internationales.
Il ne fait pas de doute que les progrès spectaculaires en génétique — de Dolly en 1997 jusqu'à la publication du génome presque complet en 2001 et à l'expérience de Séoul - remettent en question notre perception de nousmêmes en tant qu'êtres humains et nous obligent sur le plan individuel et collectif à examiner un certain nombre d'impondérables. À quel moment un être humain devient-il un être humain? Que signifie accorder la dignité humaine à un gamète, à un zygote ou à un embryon? Ces formes de vie humaine ont-elles des droits? Ces droits sont-ils supérieurs à ceux des bénéficiaires potentiels des nouvelles recherches?

La nouvelle loi présidera à la création d'un organisme pour la reproduction humaine assistée, qui aura pour tâche d'examiner les projets de recherche et d'homologuer pour la première fois toutes les cliniques de fécondité au Canada. L'homologation a pour but de prévenir l'exploitation des femmes (comme mères donneuses ou porteuses rémunérées) de manière à empêcher le commerce des dons de gamètes et à superviser l'utilisation des embryons non désirés créés in vitro à des fins de reproduction (avec les consentements appropriés) dans des recherches sur le développement de cellules souches embryonnaires. Le Code criminel prévoira des peines (dont l'emprisonnement) à l'égard des activités défendues.

Les médecins, chercheurs, chefs religieux, parlementaires et autres ont tous trouvé des imperfections dans le projet de loi C-6 et certains se sont activement opposés à son adoption. L'attention accordée aux problèmes éthiques posés par la recherche sur les cellules souches, le clonage, les mères porteuses et la criminalisation des infractions prévues dans la loi a eu pour effet de nous éloigner du but réel du projet de loi qui est d'assurer la prestation sûre, éthique et uniforme de services de reproduction.

Il ne peut y avoir d'entente parfaite sur les questions métaphysiques subsumées par la législation sur les technologies reproductives et les recherches qui les entourent. Si le projet de loi C-6 devait être bloqué au Sénat, il faudrait sans doute attendre encore plusieurs années avant qu'un gouvernement au Canada n'examine à nouveau le problème. À défaut d'une entente parfaite, notre société peut toutefois consentir à un compromis raisonnable. L'adoption du projet de loi C-6 aurait au moins pour conséquence de sortir nos chercheurs d'un vide réglementaire tout en réduisant au minimum les dangers de la reproduction humaine assistée. - $7 A M C$

\section{Référence}

1. P.C. C-6, Loi concernant la procréation assistée et la recherche connexe, $3^{\mathrm{e}}$ sess., $37^{\mathrm{e}}$ Parl, 2004. Soumis à la Chambre des communes pour $3^{\mathrm{e}}$ lecture, le 3 mars 2004 . 